Article

\title{
Effects of Microcystin-LR Exposure on Spermiogenesis in Nematode Caenorhabditis elegans
}

\author{
Yunhui Li ${ }^{1}{ }^{*}$, Minhui Zhang ${ }^{1}$, Pan Chen ${ }^{2}$, Ran Liu ${ }^{1}$, Geyu Liang ${ }^{1}$, Lihong Yin ${ }^{1}$ \\ and Yuepu Pu ${ }^{1}$
}

1 Key Laboratory of Environmental Medicine Engineering Ministry of Education, School of Public Health, Southeast University, Nanjing 210009, China;

E-Mails: zhangminhui1990@163.com (M.Z.); ranliu@seu.edu.cn (R.L.); lianggeyu@163.com (G.L.); lhyin@seu.edu.cn (L.Y.); yppu@seu.edu.cn (Y.P.)

2 Department of Molecular Pharmacology, Albert Einstein College of Medicine, Bronx, NY 10461, USA; E-Mail: pan.chen@einstein.yu.edu

* Author to whom correspondence should be addressed; E-Mail: yhli@seu.edu.cn; Tel.: +86-25-8327-2566; Fax: +86-25-8327-2583.

Academic Editor: Anna Cristina S. Samia

Received: 13 June 2015 / Accepted: 15 September 2015 /Published: 22 September 2015

\begin{abstract}
Little is known about the effect on spermiogenesis induced by microcystin-leucine arginine (MC-LR), even though such data are very important to better elucidate reproductive health. In the current work, with the aid of nematode Caenorhabditis elegans (C. elegans) as an animal model, we investigated the defects on spermiogenesis induced by MC-LR. Our results showed that MC-LR exposure induced sperm morphology abnormality and caused severe defects of sperm activation, trans-activation, sperm behavior and competition. Additionally, the expression levels of spe-15 were significantly decreased in C. elegans exposed to MC-LR lower than $16.0 \mu \mathrm{g} / \mathrm{L}$, while the expression levels of spe-10 and fer- 1 could be significantly lowered in C. elegans even exposed to $1.0 \mu \mathrm{g} / \mathrm{L}$ of MC-LR. Therefore, the present study reveals that MC-LR can induce adverse effects on spermiogenesis, and those defects of sperm functions may be induced by the decreases of spe-10, spe-15 and fer-1 gene expressions in C. elegans.
\end{abstract}

Keywords: Caenorhabditis elegans; microcystin-LR; spermiogenesis; reproductive toxicity 


\section{Introduction}

Harmful blooms of cyanobacteria occur in eutrophicated aquatic environments around worldwide and can produce a variety of toxins, including microcystins (MCs). MCs are a family of cyclic heptapeptides posing a public health threat to animals and human, among which microcystin-leucine arginine (MC-LR) is the most common and abundant variant [1,2]. MC-LR causes multiple lesions, including hepatotoxicity, neurotoxicity and kidney impairment $[3,4]$. In recent work, MC-LR has been transported rapidly to and accumulated in the gonads of mammals [5]. Animals treated with MC-LR show reduced sperm motility and concentration, and atrophy and obstruction of seminiferous tubules [6]. In addition, MC-LR exposure causes lesions of testicular Leydig cells [6], Sertoli cells [7], spermatogonia and spermatogenic cells [8]. Particularly, recent studies reported that spermatogenesis appeared to be disturbed [9] and various genes expressions associated with spermatogenesis were significantly altered [10] after MC-LR exposure. However, little information is available concerning the potential toxicity of MC-LR for male spermiogenesis.

Spermiogenesis is a complex, highly organized and regulated process. Round haploid spermatids undergo a series of morphological changes to transform into activated, mature and motility spermatozoa, which plays a very important role in the sperm transportation [11]. Errors in spermiogenesis may result in sperm abnormality that contributes to infertility [12]. However, it is difficult to detect the sperm defects stemming from impaired spermiogenesis in mammalian species $[13,14]$.

Nematode Caenorhabditis elegans (C. elegans) has been widely used as a model animal for toxicity assessment and toxicological study $[15,16]$. C. elegans has a series of features and advantages that make it a powerful tool to illuminate and study the mechanisms of reproductive toxicity of chemicals. First, C. elegans is endowed with a highly differentiated and simple reproductive system that possesses three major fundamental biological processes of gametogenesis, including mitosis, meiosis and spermiogenesis $[17,18]$. Second, $60 \%-80 \%$ of human genes are homologous with those of C. elegans, including many of conserved reproductive-related genes [19]. Third, compared with the mammalian models, in vitro spermiogenesis can be completed easily in C. elegans [20]. Fourth, C. elegans is transparent, which provides clear observation of germ cells in mature and developing nematodes [21]. Additionally, our previous study has showed that $C$. elegans is useful for assessment of reproductive toxicity relating to gametogenesis [22]. In particular, it is reported that the C. elegans assay can provide a comprehensive screening platform for assessing chemical disruption of germline function [23]. Also, C. elegans has been used as an excellent model to elucidate the germline apoptosis caused by exposure to MC-LR [24].

In this study, to assess further the MC-LR-induced toxic effects on sperm morphology and function, we utilized $C$. elegans as an animal model to explore the severe defects and the potential toxic mechanism on spermiogenesis induced by MC-LR, with the aid of sperm activation, trans-activation, and sperm behavior and competition.

\section{Results and Discussion}

Mammalian assays have indicated that MC-LR induces reproductive toxicity, although there are few reports on the effects of MC-LR exposure on spermiogenesis. This is to a large extent due to 
technical challenges in the process of spermatogenesis in mammalian assays, as well as in vitro tests [14]. Similar to the mammals, C. elegans has the conservative spermiogenesis to produce motile spermatozoons with pseudopodium instead of flagella. Sperm motility in C. elegans relies on pseudopod protrusion and retraction when hybridization occurs [25]. During the period of pseudopod extension in spermiogenesis, C. elegans spermatids can be activated in vitro by ionophore monensin, triethanolamine and Pronase [26], suggesting that activation is controlled by extracellular signals. Male-derived sperm activators are also involved in the process of pseudopod extension in spermiogenesis [27]. Therefore, we investigated pseudopod extension controlled by extracellular signals, activators of male-derived sperm determined by assays of sperm activation and trans-activation in spermiogenesis. Exposure to MC-LR increased the number of abnormal activated sperm with short pseudopods and reduced the percentage of activated sperm (Figure 1a). The inhibition of sperm activation suggested that MC-LR might damage the sperm extracellular signals. Sperm trans-activation was also performed to observe that male-derived activators transactivated hermaphrodite-derived sperm to produce hermaphrodite-derived progeny (self-progeny). The result showed that the number of self-progeny in C. elegans was decreased when exposed to $64.0 \mu \mathrm{g} / \mathrm{L}$ (Figure 1b). The decline of sperm transactivation suggested that MC-LR might damage male-derived sperm activators. We further found that spermatids morphology was significantly altered following MC-LR exposure (Figure 2), while spermatids diameter and cross-sectional area in C. elegans showed nonsignificant changes compared with the control groups (Figure 3). Therefore, we suspected that MC-LR suppressed sperm activation and transactivation, which may result directly in abnormalities in spermatids morphology and pseudopod extension. This suggests that these defects in C. elegans exposed to MC-LR may be largely related to spermiogenesis.
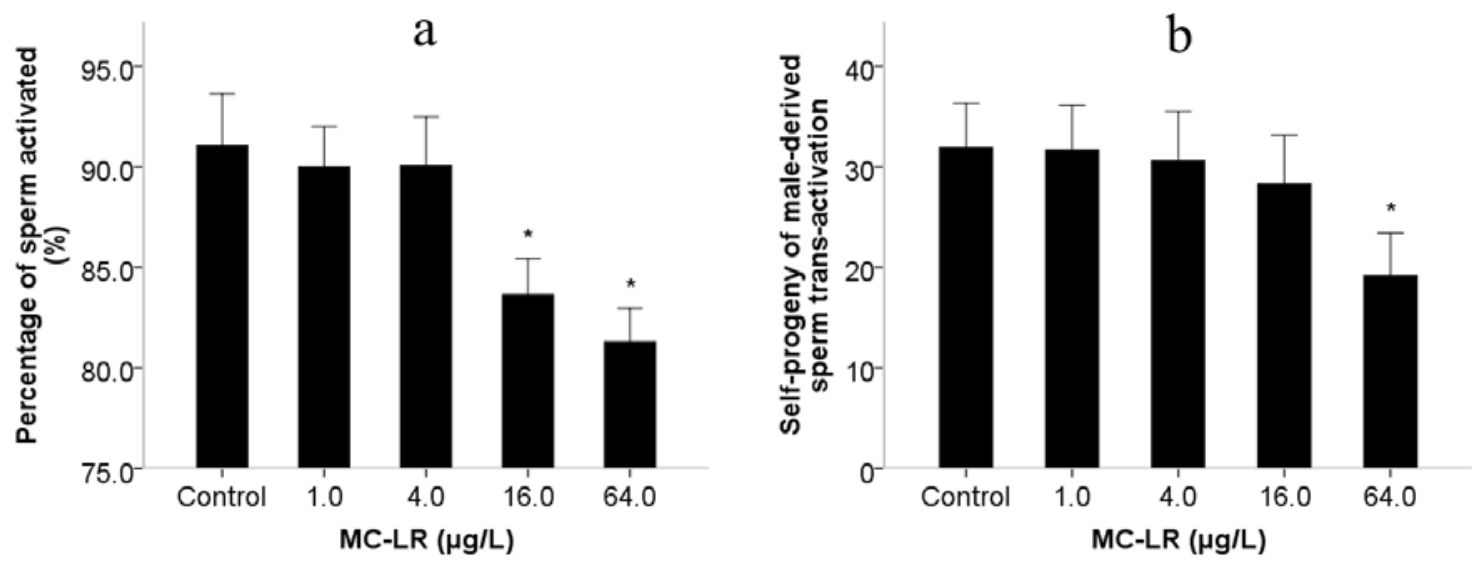

Figure 1. Effects of microcystin-leucine arginine (MC-LR) exposure on nematodes sperm activation (a) him-5 sperm activation in vitro with Pronase; and (b) Number of self-progeny of male-derived sperm trans-activation. Bars represent means $\pm \mathrm{SEM}$. ${ }^{*} p<0.05$ vs. the control group. 


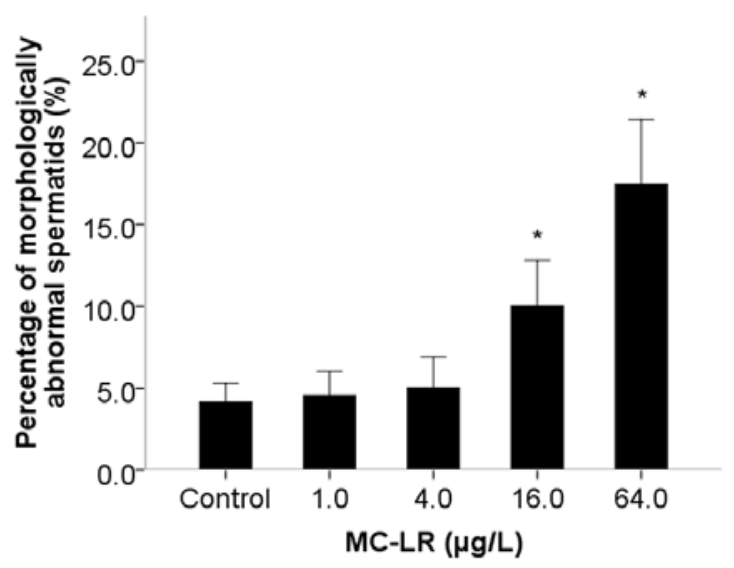

Figure 2. Effects of MC-LR exposure on spermatids morphology. Bars represent means \pm SEM. $* p<0.05$ vs. the control group.
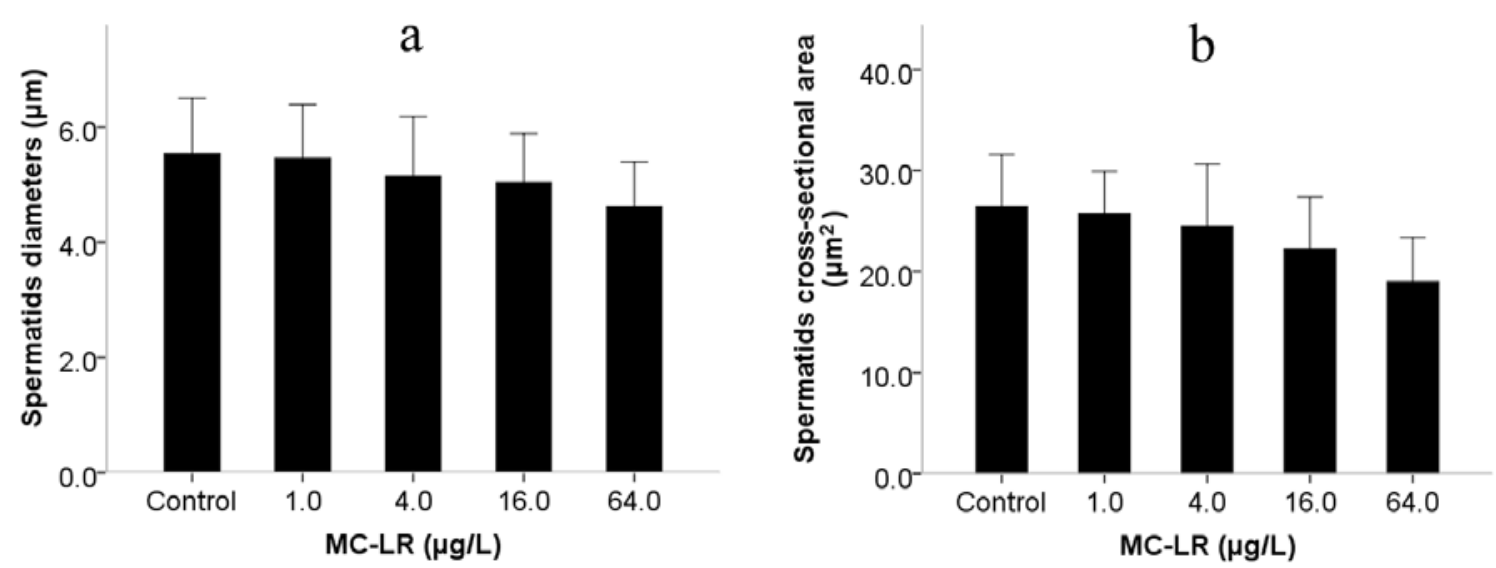

Figure 3. Effects of MC-LR exposure on spermatids size. (a) Spermatids diameters; and (b) Spermatids cross-sectional area. One male was dissected and 200 spermatids were analyzed. Bars represent means \pm SEM.

During the period of male mated with female, male-derived sperm are activated after ejaculation into the female reproductive tract and targeted for spermathecae. MitoTracker Red (Invitrogen, Carlsbad, CA, USA) was used to visualize male-derived sperm migration in female reproductive tract. It suggested that sperm migration is suppressed if the mitotracker-labeled sperms are seen in ectopic positions such as uterus and vulva in female [27,28]. Our results showed that sperm migration of C. elegans was suppressed by exposure to MC-LR at a dose of $64.0 \mu \mathrm{g} / \mathrm{L}$ (Figure 4). Further, we also investigated the ability of male-derived sperm competition through accounting the numbers of self progeny and outcross progeny. Compared with the 33\% self-progeny percentage in the control group, the percentage with $64.0 \mu \mathrm{g} / \mathrm{L} \mathrm{MC-LR}$ was significantly increased to $43 \%$ ( $p<0.05$ ) (Figure 5). Previous studies indicated that sperm competitiveness was strongly correlated with sperm size [29], while our results showed no change in spermatids diameters and cross-sectional area. This implies that MC-LR might inhibit sperm competitiveness by other unidentified mechanisms. We used C. elegans as a model to bring new insights into the reproductive effects of MC-LR by providing the first evidence 
that MC-LR exposure can result in defects of spermiogenesis, including extracellular signals and auto-activators of sperm activation, sperm behavior and competitiveness.



Figure 4. Effects of MC-LR exposure on sperm migration. The number of abnormal sperm migration worms and total worms were counted. Bars represent means \pm SEM. ${ }^{*} p<0.05 v s$. the control group.

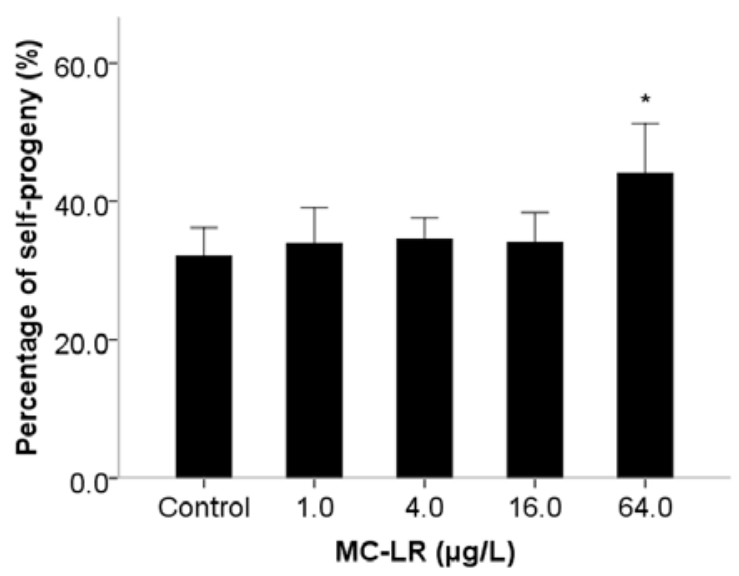

Figure 5. Effects of MC-LR exposure on sperm competitiveness. The number of self-progeny and outcrossed-progeny were counted. Bars represent means \pm SEM. ${ }^{*} p<0.05$ vs. the control group.

Based on the mentioned impairments of MC-LR, the expression levels of four genes (spe-10, spe-15, fer-1 and folt-1) were analyzed to explore the potential molecular mechanisms. spe-10 is required for spermatids development in spermatogenesis and fertility [30], while spe-15 is related to spermiogenesis or sperm activation [31]. fer-1 plays a role in sperm pseudopods extension and motility [32]. folt-1 is related to germline function and sperm count [33,34]. Besides, spe-10, spe-15 and fer-1are involved in the transportation of sperm fibrous body-membranous organelles (FB-MOs) and FBs disassemble, respectively. Therefore, the three genes were selected based on the continuous development of FB-MOs, which is important for spermatogenesis [18]. We speculated that MC-LR might damage spermiogenesis by disrupting the development of FB-MOs, although the more serious decrease in expression of three genes was not completely consistent with the toxic changes of spermiogenesis (Figure 6). A particularly interesting feature is that we did not observe any change in folt-1 expression 
level (Figure 6), although MC-LR decreased sperm numbers in C. elegans (data not shown). Therefore, the results imply that folt- 1 is not involved in the regulation of MC-LR-induced spermiogenesis defects. Other genes related to signaling pathways require further exploration.

A previous study reported that spermatogenesis is disturbed by exposure to MC-LR [9]. Here, we extended this observation by providing evidence that MC-LR induced spermiogenesis defects and subsequent process abnormality until fertilization of C. elegans. Our results also show that C. elegans, as a simple and reliable model organism, has a unique advantage in studying the process of spermatogenesis and may complement in vitro and whole-organism assays in reproductive toxicology.
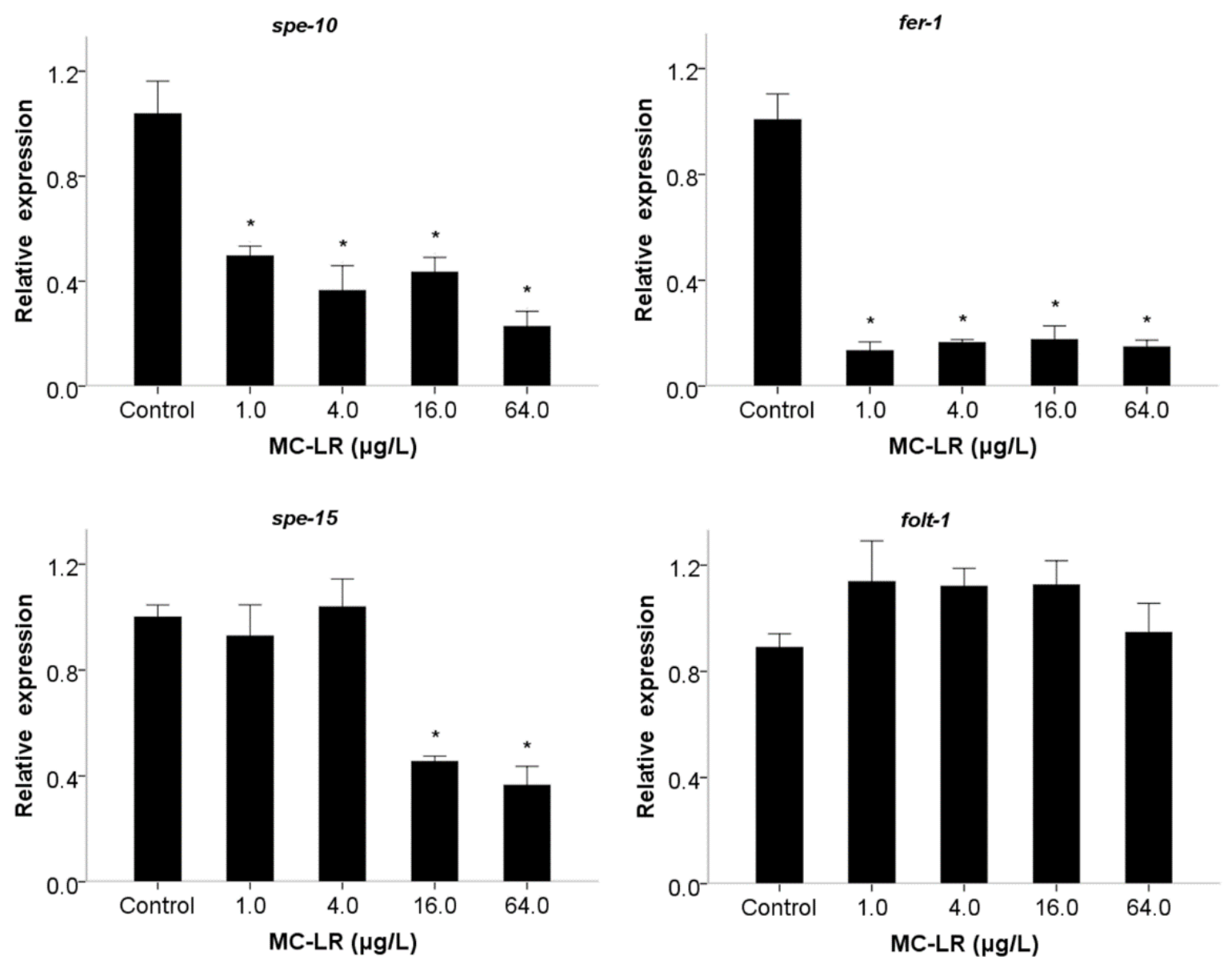

Figure 6. Effects of MC-LR exposure on relative mRNA expression levels of genes involved in spermiogenesis. Bars represent means \pm SEM. ${ }^{*} p<0.01$ vs. the control group.

\section{Experimental Section}

The strains used in this study were wild-type N2, him-5(e1490), fog-2(q71), dpy-5(e61), fer-1(hc1); him-5(e1490) and spe-8(hc40); $d p y-4(e 1166)$, originally obtained from the Caenorhabditis Genetics Center (CGC, University of Minnesota, Minneapolis, MN, USA). Nematodes were grown and maintained at $20^{\circ} \mathrm{C}$ as described [21]. Gravid nematodes were removed OP50 by M9 buffer and eggs were harvested by a bleaching mixture $(0.45 \mathrm{M} \mathrm{NaOH}, 2 \% \mathrm{HOCl})$. Age-synchronized populations of L4-larvae nematodes were obtained by the collection cultured in $20^{\circ} \mathrm{C}$. 
MC-LR (purity $\geq 95 \%$ ) was purchased from Alexis Biochemicals Corporation (Lausen, Switzerland). MC-LR (1 mg) was dissolved in $1 \mathrm{~mL}$ of methanol and diluted to $1000 \mu \mathrm{g} / \mathrm{L}$ with M9 buffer to prepare the stock solution. The concentrations of MC-LR of working solutions were analyzed by Agilent 1100 liquid chromatograph (Agilent Technologies, Santa Clara, CA, USA). The HPLC conditions were performed in our previous study as description [35]. MC-LR exposure was performed according to the agar toxicity test as described [36]. The final MC-LR concentrations of culture medium were 1.0, 4.0, 16.0 and $64.0 \mu \mathrm{g} / \mathrm{L}$, respectively. The nematodes were exposed to MC-LR for $48-\mathrm{h}$ at $20^{\circ} \mathrm{C}$ and the solvent controls were prepared by the same way.

The sperm activation was performed according to previous description [37]. him-5 male was dissected to release sperm into SM buffer containing $10 \mu \mathrm{L}$ Pronase E $(200 \mu \mathrm{g} / \mathrm{mL})$. The percentage of activated sperm was calculated as follows: activated sperm/total sperm $\times 100.10$ nematodes were used in control and exposed groups, and three replicates were performed.

Four virgin fer-1; him-5 males were crossed at the ratio of 4:1 with one spe-8; dpy-4 hermaphrodite to analyze the transactivation as described [38]. Each plate was considered a single mating trial, and ten plates were scored. Only mating successful hermaphrodites were transferred to the new NGM plate, respectively. Progeny were scored until the hermaphrodites were no eggs lying. Three replicates were performed.

The sperm size and morphology were measured and analyzed as previously published [22], him-5 male was dissected to release spermatids. Five different visual fields about 200 spermatids for each sample were captured under a differential interference contrast (DIC) microscope (Olympus BX41, Olympus, Tokyo, Japan) [39]. The spermatids diameter, cross-sectional area and morphology were measured and analyzed using soft image-pro plus 6.0 (Media Cybernetics, Rockville, MD, USA). 10 nematodes were used and three replicates were performed.

To analyze the mitotracker sperm migration, observation of the mitotracker-labeled male-derived sperms movement within female reproductive tracts was based on previous description [28]. Synchronized him-5 males were labeled and incubated in both MitoTracker Red CMXRos (Invitrogen, Carlsbad, CA, USA) and MC-LR solution for 48-h at $20^{\circ} \mathrm{C}$, then crossed at the ratio of 3:1 with young adult of fog-2 female for 8-h. Then female was transferred to a new NGM plate for 2-h ensuring enough time for sperms migrating to the spermatheca before the microscopy observation. Mitotracker fluorescence was observed by fluorescence microscopy (Olympus FSX100, Olympus, Tokyo, Japan). Only mating successful and corpse integrity worms were practical. The abnormal sperms migration were seen in ectopic positions such as uterus and vulva in female. The percentage of abnormal sperm migration (abnormal migration worms/total worms $\times 100$ ) was calculated. 10 nematodes were used and three replicates were performed.

The sperm competition was performed as previously described [40]. L4 him-5 males were exposed to MC-LR for 48-h, then crossed with $d p y-5$ hermaphrodites (4:1) for 24 -h at $20{ }^{\circ} \mathrm{C}$. Ten successful mating trails were tested. The number of short-limb phenotype (self-progeny) and normal-limb phenotype (outcrossed-progeny) were counted. Three replicates were performed.

To determine C. elegans spermiogenesis relative genes expression levels, real-time Quantitative PCR (qRT-PCR) was performed to measure the reverse transcription products using SYBR Green I dye (TOYOBO, Osaka, Japan). The primers were performed in Table 1. Relative expression levels were determined with Mastercycler gradient PCR (Eppendorf, Hamburg, Germany) and ABI 7300 
Quantitative PCR (ABI, Carlsbad, CA, USA). Relative RNA expression levels were determined using the $2^{-\Delta \Delta C t}$ method with three replicates for each group [22].

Table 1. Gene primers tested in the study.

\begin{tabular}{|c|c|c|c|}
\hline \multicolumn{2}{|c|}{ Gene Primer } & \multirow{3}{*}{$\begin{array}{c}\text { Sequence } \\
\text { TTTTATTGTCGGCGGAGTGT } \\
\text { CGATGACTGCGAACTTTGAG }\end{array}$} & \multirow{3}{*}{$\begin{array}{c}\text { Annealing Temperature } \\
57.8^{\circ} \mathrm{C}\end{array}$} \\
\hline \multirow{2}{*}{ spe-10 } & Forward & & \\
\hline & Reverse & & \\
\hline \multirow{2}{*}{ spe-15 } & Forward & GGAGTTTTGGATGTCGCTGGTT & \multirow{2}{*}{$60.4^{\circ} \mathrm{C}$} \\
\hline & Reverse & GCTCTCTGGGTGAAATGTTGGA & \\
\hline \multirow{2}{*}{ fer-1 } & Forward & AATGGATGGAATGCTGTTGGTC & \multirow{2}{*}{$57.8^{\circ} \mathrm{C}$} \\
\hline & Reverse & AACGCTTTCTGAAGTTGTGGTG & \\
\hline \multirow{2}{*}{ folt-1 } & Forward & TCCATTCCTCACTCCGTTTCTA & \multirow{2}{*}{$60.4^{\circ} \mathrm{C}$} \\
\hline & Reverse & GCATCTGCCATACTCСТTTACC & \\
\hline \multirow{2}{*}{$a c t-1$} & Forward & ATGTGTGACGACGAGGTT & \multirow{2}{*}{$60.4^{\circ} \mathrm{C}$} \\
\hline & Reverse & GAAGCACTTGCGGTGAAC & \\
\hline
\end{tabular}

Data were plotted as means \pm Standard Error of the Mean (SEM). One-way ANOVA/Dunnett's $t$-test was used for comparison between the control and the exposed groups. Probability levels of 0.05 and 0.01 were considered statistically significant. Statistical analysis was processed using SPSS 13.0 (SPSS Inc., Chicago, IL, USA).

\section{Conclusions}

In our current works, we found that spe-10, spe-15, and fer-1 gene are involved in mediating MC-LR-induced sperm extracellular signals and sperm own activators abnormalities, and this could result in sperm abnormal pseudopods. Those abnormalities were effected together to induce sperm morphological defects of the activation process in spermiogenesis and further inhibit sperm migration, eventually lead to sperm competitiveness decline. Although the study showed a potential signal transduction pathway in modulating MC-LR-induced spermiogenesis abnormality, more related mechanisms should be further explored.

\section{Acknowledgments}

We declared that the experiments comply with the current laws of China. The nematode strains used in this study were provided by the Caenorhabditis Genetics Center (funded by the NIH National Center for Research Resource, USA). This work was supported by National Natural Science Foundation of China (Nos. 81273123, 81172618) and 333 Project of Jiangsu Province (No. 2012).

\section{Author Contributions}

Yunhui Li conceived, designed and performed the experiments, as well as wrote the manuscript; Minhui Zhang performed the experiments and analyzed the data; Pan Chen conceived and revised the manuscript; Ran Liu and Geyu Liang contributed reagents and materials, and analyzed the data; Yuepu Pu and Lihong Yin were involved in conceiving the experiments and took part in preparation of the manuscript. 


\section{Conflicts of Interest}

The authors declare no conflict of interest.

\section{References}

1. Carmichael, W.W.; Azevedo, S.M.; An, J.S.; Molica, R.J.; Jochimsen, E.M.; Lau, S.; Rinehart, K.L.; Shaw, G.R.; Eaglesham, G.K. Human fatalities from cyanobacteria: Chemical and biological evidence for cyanotoxins. Environ. Health Perspect. 2001, 109, 663-668.

2. Codd, G.A.; Morrison, L.F.; Metcalf, J.S. Cyanobacterial toxins: Risk management for health protection. Toxicol. Appl. Pharmacol. 2005, 203, 264-272.

3. Vasconcelos, V.M.; Sivonen, K.; Evans, W.R.; Carmichael, W.W.; Namikoshi, M. Hepatotoxic microcystin diversity in cyanobacterial blooms collected in Portuguese freshwaters. Water Res. 1996, 30, 2377-2384.

4. Maidana, M.; Carlis, V.; Galhardi, F.G.; Yunes, J.S.; Geracitano, L.A.; Monserrat, J.M.; Barros, D.M. Effects of microcystins over short- and long-term memory and oxidative stress generation in hippocampus of rats. Chem. Biol. Interact. 2006, 159, 223-234.

5. Wang, Q.; Xie, P.; Chen, J.; Liang, G. Distribution of microcystins in various organs (heart, liver, intestine, gonad, brain, kidney and lung) of Wistar rat via intravenous injection. Toxicon 2008, 52, 721-727.

6. Li, Y.; Sheng, J.; Sha, J.; Han, X. The toxic effects of microcystin-LR on the reproductive system of male rats in vivo and in vitro. Reprod. Toxicol. 2008, 26, 239-245.

7. Li, Y.; Han, X. Microcystin-LR causes cytotoxicity effects in rat testicular Sertoli cells. Environ. Toxicol. Pharmacol. 2012, 33, 318-326.

8. Zhou, Y.; Chen, Y.; Yuan, M.; Xiang, Z.; Han, X. In vivo study on the effects of microcystin-LR on the apoptosis; proliferation and differentiation of rat testicular spermatogenic cells of male rats injected i.p. with toxins. J. Toxicol. Sci. 2013, 38, 661-670.

9. Trinchet, I.; Djediat, C.; Huet, H.; Dao, S.P.; Edery, M. Pathological modifications following sub-chronic exposure of medaka fish (Oryzias latipes) to microcystin-LR. Reprod. Toxicol. 2011, 32, 329-340.

10. Zhou, Y.; Xiang, Z.; Li, D.; Han, X. Regulation of microcystin-LR-induced toxicity in mouse spermatogonia by miR-96. Environ. Sci. Technol. 2014, 48, 6383-6390.

11. Hermo, L.; Pelletier, R.M.; Cyr, D.G.; Smith, C.E. Surfing the wave, cycle, life history, and genes/proteins expressed by testicular germ cells. Part 1: Background to spermatogenesis, spermatogonia, and spermatocytes. Microsc. Res. Tech. 2010, 73, 241-278.

12. Chemes, H.E.; Rawe, V.Y. The making of abnormal spermatozoa: Cellular and molecular mechanisms underlying pathological spermiogenesis. Cell Tissue Res. 2010, 341, 349-357.

13. Muller, C.H. Rationale, interpretation, andrology lab corner validation, and uses of sperm function tests. J. Androl. 2000, 21, 10-30.

14. Cheng, C.Y.; Mruk, D.D. Regulation of spermiogenesis, spermiation and blood-testis barrier dynamics: Novel insights from studies on Eps8 and Arp3. Biochem. J. 2011, 435, 553-562. 
15. Leung, M.C.; Williams, P.L.; Benedetto, A.; Au, C.; Helmcke, K.J.; Aschner, M.; Meyer, J.N. Caenorhabditis elegans: An emerging model in biomedical and environmental toxicology. Toxicol. Sci. 2008, 106, 5-28.

16. Huguier, P.; Manier, N.; Meline, C.; Bauda, P.; Pandard, P. Improvement of the Caenorhabditis elegans growth and reproduction test to assess the ecotoxicity of soils and complex matrices. Environ. Toxicol. Chem. 2013, 32, 2100-2108.

17. Lesch, B.J.; Page, D.C. Genetics of germ cell development. Nat. Rev. Genet. 2012, 13, 781-794.

18. Chu, D.S.; Shakes, D.C. Spermatogenesis. In Germ Cell Development in C. elegans; Springer: New York, NY, USA, 2013; Volume 757, pp. 171-203.

19. Kaletta, T.; Hengartner, M.O. Finding function in novel targets: C. elegans as a model organism. Nat. Rev. Drug Discov. 2006, 5, 387-398.

20. Ward, S.; Carrel, J.S. Fertilization and sperm competition in the nematode Caenorhabditis elegans. Dev. Biol. 1979, 73, 304-321.

21. Brenner, S. The genetics of Caenorhabditis elegans. Genetics 1974, 77, 71-94.

22. Ruan, Q.L.; Ju, J.J.; Li, Y.H.; Li, X.B.; Liu, R.; Liang, G.Y.; Zhang, J.; Pu, Y.P.; Wang, D.Y.; Yin, L.H. Chlorpyrifos exposure reduces reproductive capacity owing to a damaging effect on gametogenesis in the nematode Caenorhabditis elegans. J. Appl. Toxicol. 2012, 32, 527-535.

23. Allard, P.; Kleinstreuer, N.C.; Knudsen, T.B.; Colaiacovo, M.P.A. C. elegans screening platform for the rapid assessment of chemical disruption of germline function. Environ. Health Perspect. 2013, 121, 717-724.

24. Wang, S.C.; Geng, Z.Z.; Wang, Y.; Tong, Z.H.; Yu, H.Q. Essential roles of p53 and MAPK cascades in microcystin-LR-induced germline apoptosis in Caenorhabditis elegans. Environ. Sci. Technol. 2012, 46, 3442-3448.

25. Ma, X.; Zhao, Y.; Sun, W.; Shimabukuro, K.; Miao, L. Transformation: How do nematode sperm become activated and crawl? Protein Cell 2012, 3, 755-761.

26. Shakes, D.C.; Ward, S. Initiation of spermiogenesis in C. elegans: A pharmacological and genetic analysis. Dev. Biol. 1989, 134, 189-200.

27. Geldziler, B.; Chatterjee, I.; Singson, A. The genetic and molecular analysis of spe-19, a gene required for sperm activation in Caenorhabditis elegans. Dev. Biol. 2005, 283, 424-436.

28. Kubagawa, H.M.; Watts, J.L.; Corrigan, C.; Edmonds, J.W.; Sztul, E.; Browse, J.; Miller, M.A. Oocyte signals derived from polyunsaturated fatty acids control sperm recruitment in vivo. Nat. Cell Biol. 2006, 8, 1143-1148.

29. LaMunyon, C.W.; Ward, S. Larger sperm outcompete smaller sperm in the nematode Caenorhabditis elegans. Proc. R. Soc. Lond. B: Biol. Sci. 1998, 265, 1997-2002.

30. Gleason, E.J.; Lindsey, W.C.; Kroft, T.L.; Singson, A.W.; L’Hernault, S.W. spe-10 encodes a DHHC-CRD zinc-finger membrane protein required for endoplasmic reticulum/Golgi membrane morphogenesis during Caenorhabditis elegans spermatogenesis. Genetics 2006, 172, 145-158.

31. Kelleher, J.F.; Mandell, M.A.; Moulder, G.; Hill, K.L.; L’Hernault, S.W.; Barstead, R.; Titus, M.A. Myosin VI is required for asymmetric segregation of cellular components during C. elegans spermatogenesis. Curr. Biol. 2000, 10, 1489-1496. 
32. Krajacic, P.; Hermanowski, J.; Lozynska, O.; Khurana, T.S.; Lamitina, T. C. elegans dysferlin homolog fer-1 is expressed in muscle, and fer-1 mutations initiate altered gene expression of muscle enriched genes. Physiol. Genom. 2009, 40, 8-14.

33. Balamurugan, K.; Ashokkumar, B.; Moussaif, M.; Sze, J.Y.; Said, H.M. Cloning and functional characterization of a folate transporter from the nematode Caenorhabditis elegans. Am. J. Physiol. Cell Physiol. 2007, 293, C670-C681.

34. Austin, M.U.; Liau, W.S.; Balamurugan, K.; Ashokkumar, B.; Said, H.M.; LaMunyon, C.W. Knockout of the folate transporter folt-1 causes germline and somatic defects in C. elegans. BMC Dev. Biol. 2010, 10, doi:10.1186/1471-213X-10-46.

35. Ju, J.; Ruan, Q.; Li, X.; Liu, R.; Li, Y.; Pu, Y.; Yin, L.; Wang, D. Neurotoxicological evaluation of microcystin-LR exposure at environmental relevant concentrations on nematode Caenorhabditis elegans. Environ. Sci. Pollut. Res. 2013, 20, 1823-1830.

36. Anderson, G.L.; Boyd, W.A.; Williams, P.L. Assessment of sublethal endpoints for toxicity testing with the nematode Caenorhabditis elegans. Environ. Toxicol. Chem. 2001, 20, 833-838.

37. Singaravelu, G.; Chatterjee, I.; Marcello, M.R.; Singson, A. Isolation and in vitro activation of Caenorhabditis elegans sperm. Annu. Rev. Cell Dev. Biol. 2007, 23, 405-433.

38. Achanzar, W.E.; Ward, S. A nematode gene required for sperm vesicle fusion. J. Cell Sci. 1997, 110, 1073-1081.

39. L'Hernault, S.W.; Roberts, T.M. Cell biology of nematode sperm. Methods Cell Biol. 1995, 48, 273-301.

40. Singson, A.; Hill, K.L.; L'Hernault, S.W. Sperm competition in the absence of fertilization in Caenorhabditis elegans. Genetics 1999, 152, 201-208.

(C) 2015 by the authors; licensee MDPI, Basel, Switzerland. This article is an open access article distributed under the terms and conditions of the Creative Commons Attribution license (http://creativecommons.org/licenses/by/4.0/). 